\title{
Hypotrichosis with juvenile macular dystrophy: Portuguese case
}

\section{Fatima Zahra Elfatoiki ${ }^{1}$, Florance Cordoliani', Pascal Regane ${ }^{2}$, Aude Affortit-Demoge ${ }^{3}$, Michel Rybojad ${ }^{4}$}

${ }^{1}$ Université Paris Diderot, Sorbonne Paris Cité, AP-HP, Service de Dermatologie, Hôpital Saint Louis, Paris, France, ${ }^{2}$ Université Paris Diderot, Sorbonne Paris Cité, AP-HP, Centre de Sabouraud, Paris, France, ${ }^{3}$ Service d'Ophtalmologie, Fondation Ophtalmologique Adolphe de Rothschild, Paris, France, ${ }^{4}$ Université Paris Diderot, Sorbonne Paris Cité, AP-HP, Service de Dermatologie, Hôpital Saint Louis, Paris, France

Corresponding author: Dr. Fatima Zahra Elfatoiki, E-mail: Fatiza59@hotmail.fr

\begin{abstract}
Hypotrichosis with juvenile macular dystrophy is a rare congenital disease mainly found in Druze population of Northern Israel. This disorder caused by CDH3 mutation encoding

P-cadherin, which is expressed in retinal pigment epithelium and hair follicles. A 11 -year-old girl was born to related Portuguese parents, had hypotrychosis since birth and macular dystrophy diagnosed at age 5 . Fundus examination and fluorescein angiography revealed located macular pigmentary abnormalities. No molecular analysis was done. Fundus examination should be considered as mandatory in the assessment of congenital hypotrichosis.
\end{abstract}

Key words: Macular dystrophy; Hypotrichosis; Congenital disease; CDH3 mutation, P-cadherin

\section{INTRODUCTION}

Hypotrichosis with juvenile macular dystrophy is a rare autosomal recessive disorder, mainly described in Israeli families of Arab Muslim origin. We report a case of this disease in portuguese family.

\section{CASE REPORT}

A 11-year-old girl, the second daughter of third-degree consanguineous parents who were from Portugal. She was seen in our department for short and slow-growing hair present since birth without sweating abnormality. Her past medical history included macular dystrophy diagnosed at age 5 without any visual impact. There was no family history of hair or visual impairment and her own brother was unaffected.

Physical examination revealed hypotrychosis, sparse short hairs over the entire scalp without alopecia or erythema. The hair pull test was negative. The eyebrows and eyelashes were normal and no abnormalities of skin, teeth or nails were noted. General examination of the respiratory, cardiovascular and central nervous systems did not show abnormalities.

A complete blood count was normal. The blood iron, ferritin, vitamin B12, and thyroid hormone levels were all within normal ranges.

Trichogram analysis demonstrated a reduced diameter of hair and polarized light examination showed non specific hair dystrophy. Fundus examination and fluorescein angiography revealed located macular pigmentary abnormalities. Visual acuity and ERG testing were normal. Molecular analysis of the $\mathrm{CDH} 3$ gene was not done.

\section{DISCUSSION}

Hypotrichosis with juvenile macular dystrophy is a rare autosomal recessive disorder. It has been firstly described in 1935 by Wagner in two German

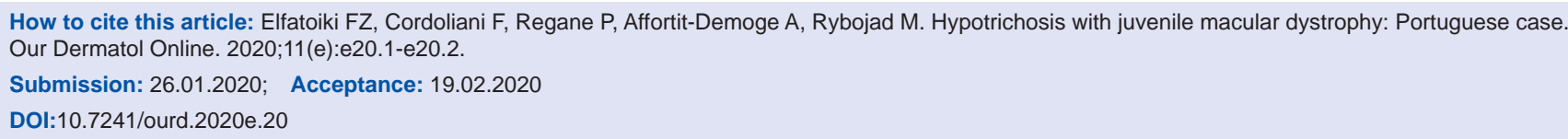


sisters [1]. It characterized by hypotrichosis and progressive macular dystrophy leading to blindness. Hair abnormalities include short and sparse scalp hair, which apparently results from an increased proportion of involuting hair follicles, thus alluding to a cell cycle defect. Various forms of macular dystrophies have been reported, including hypopigmentation, mild pigmentary changes and severe hyperpigmentation [2]. While hair changes are stable, the retinal disorder is progressive, affecting cone-mediated as well as rodmediated vision, and causing decreased visual acuity and early blindness between the second and the fourth decades of life [3].

Hypotrichosis with juvenile macular dystrophy is caused by $\mathrm{CDH} 3$ mutation. The $\mathrm{CDH} 3$ gene locus encodes P-cadherin, a classical cadherin molecule and a major component of the adherens junction in several tissues including retinal pigment epithelium and hair follicles [4].

The disease was shown in families of various origins and mainly found in Israeli families of Arab Muslim origin. Those families belonged to the Druze population who had lived in mountainous areas of the Middle East as a closed society almost from their inception in Cairo around 1017 A.D [3,5]. Several CDH3 mutations have been described causing this disease, the missense $\mathrm{R} 503 \mathrm{H}$ mutation was reported to be the most common CDH3 mutation in the Muslim Arab Israeli population [3].

Our patient was born to related Portuguese parents. Two other Portuguese cases had been previously described in two siblings in 1995. The parents of these patients were not consanguineous and were native of the same village. They had abnormally sparse hair and at the age of 25 years had still never cut their hair. Ophthalmologic examination revealed reduced visual acuity and macular pigmentary degenerative changes in both of them. No molecular analysis was done [6].

Earlier identification of hypotrichosis with juvenile macular dystrophy patients allows for educational measures to be taken, appropriate counselling and visual rehabilitation to be provided. Fundus examination should be considered as mandatory in the assessment of congenital hypotrichosis.

\section{Consent}

The examination of the patient was conducted according to the Declaration of Helsinki principles.

The authors certify that they have obtained all appropriate patient consent forms. In the form the patient(s) has/have given his/her/their consent for his/ her/their images and other clinical information to be reported in the journal. The patients understand that their names and initials will not be published and due efforts will be made to conceal their identity, but anonymity cannot be guaranteed.

\section{REFERENCES}

1. Wagner H. Makulaaffektion vergesellschaftet mit Haarabnormalitat von anugotypus, beide vielleicht angeboren bei zwei Geschwistern. Graefes Arch Klin Exp Ophthalmol. 1935;134:71.

2. Indelman M, Leibu R, Jammal A, Bergman R, Sprecher E. Molecular basis of hypotrichosis with juvenile macular dystrophy in two siblings. Br J Dermatol. 2005;153:635-8.

3. Avitan-Hersh E, Indelman M, Khamaysi Z, Leibu R, Bergman R. A novel nonsense $\mathrm{CDH} 3$ mutation in hypotrichosis with juvenile macular dystrophy. Int J Dermatol. 2012;51:325-7.

4. Shimomura Y, Wajid M, Kurban M, Christiano AM. Splice site mutations in the P-cadherin gene underlie hypotrichosis with juvenile macular dystrophy. Dermatology. 2010;220:208-12.

5. Sprecher E, Bergman R, Richard G, Lurie R, Shalev S, Petronius D, et al. Hypotrichosis with juvenile macular dystrophy is caused by a mutation in $\mathrm{CDH} 3$, encoding P-cadherin. Nat Genet. 2001;29:134-6.

6. Souied E, Amalric P, Chauvet ML, Chevallier C, Le Hoang P, Munnich A, et al. Unusual association of juvenile macular dystrophy with congenital hypotrichosis: occurrence in two siblings suggesting autosomal recessive inheritance. Ophthalmic Genet. 1995;16:11-5.

Copyright by Fatima Zahra Elfatoiki, et al. This is an open-access article distributed under the terms of the Creative Commons Attribution License, which permits unrestricted use, distribution, and reproduction in any medium, provided the original author and source are credited.

Source of Support: Nil, Conflict of Interest: None declared. 